\title{
GENUS GROUP OF FINITE GALOIS EXTENSIONS
}

\author{
TERUO TAKEUCHI
}

\begin{abstract}
Let $K / k$ be a Galois extension of finite degree, and let $K^{\prime}$ denote the maximal abelian extension over $k$ contained in the Hilbert class field of $K$. We give formulas about the group structure of $\mathrm{Gal}\left(K^{\prime} / k\right)$ and the genus group of $K / k$, which refine the ordinary genus formula.
\end{abstract}

For an algebraic number field $k$ of finite degree, let $\mathrm{Cl}(k)$ denote the ideal class group of $k$. For a modulus $S$ of $k$ (i.e., a finite product of primes of $k$ ), let $I_{k}(S)$, $P_{k}(S)$, and $P_{k S}$ denote the group of ideals of $k$ prime to $S$, the group of principal ideals of $k$ prime to $S$, and the ray ideal group modulo $S$ in $k$, respectively. Similarly, let $k(S)$ and $k_{S}$ denote the group of elements of $k$ prime to $S$ and the ray number group of $k$ modulo $S$, respectively. Let $K / k$ be a Galois extension of finite degree. Let $K^{\prime}$ be the maximal abelian extension over $k$ contained in the Hilbert class field $\bar{K}$ of $K$. Then, by definition, the genus field $K^{*}$ of $K / k$ in the wide sense is $K \cdot K^{\prime}$, and the genus group of $K / k$ is $\operatorname{Gal}\left(K^{*} / K\right)$.

The following lemma is well known and proved by a standard manner in class field theory.

LEMMA 1. Let $\mathfrak{f}^{\prime}$ be the conductor of $K^{\prime} / k$; then $K^{\prime}$ is a class field over $k$ corresponding to $I_{k}\left(f^{\prime}\right) / N_{K / k}\left(P_{K}\left(f^{\prime}\right)\right) P_{k f^{\prime}}$. Moreover, if $K / k$ is abelian, then $f^{\prime}$ coincides with the conductor of $K / k$.

For a modulus $f$ with $\mathfrak{f}^{\prime} \mid \mathfrak{f}$, put $\mathscr{S}(K / k)=I_{k}(\mathfrak{f}) / N_{K / k}\left(P_{K}(\mathfrak{f})\right) P_{k \mathfrak{f}}$. Clearly, (S) $(K / k)$ does not depend on the choice of such $f$ (up to isomorphisms). The purpose of this paper is to describe the $l^{i}$-rank of $\mathbb{S}(K / k)$. Let $l$ be a prime number. Throughout this paper we fix $l$ unless otherwise stated. For an abelian group $A$ written multiplicatively, let $\operatorname{rank}_{i}(A)$ denote the $l^{i}$-rank of $A$, i.e., the $F_{1}$-dimension of $A^{l^{i-1}} / A^{l^{i}}$. For $i \geqslant 0$, put $F_{i}=\left\{a \in k^{\times} \mid(a) \in P_{k}^{l^{i}}\right\}$. Then $k^{\times} \supset F_{1}$ $\supset F_{2} \supset \cdots \supset F_{i} \supset \cdots \supset E_{k}$ and $F_{i} \supset F_{i-1}^{l} E_{k}$, where $E_{k}$ denotes the group of units in $k$. Put $F_{i}(S)=F_{i} \cap k(S)$.

LEMMA 2. Let $1 \rightarrow N \rightarrow M \rightarrow L \rightarrow 1(N \subset M)$ be an exact sequence of finite abelian groups. Put $N_{i}=N \cap M^{i^{i}}$. Then for $i \geqslant 1$, we have

$$
\begin{aligned}
\operatorname{rank}_{i}(M) & =\operatorname{rank}_{i}(L)+\operatorname{rank}_{1}\left(N_{i-1} / N_{i}\right) \\
& =\operatorname{rank}_{i}(L)+\log _{l}\left\{\#\left(N_{i-1} / N_{i}\right)\right\} .
\end{aligned}
$$

Received by the editors October 11, 1985.

1980 Mathematics Subject Classification (1985 Revision). Primary 11R37, 11R65. 
Proo'f. By the definition of $l^{i}$-rank, we see $\operatorname{rank}_{i}(L)=\operatorname{rank}_{1}\left(L^{l^{i-1}} / L^{l^{i}}\right)=$ $\operatorname{rank}_{1}\left(M^{l^{i-1}} N / M^{l^{i}} N\right)$. On the other hand,

$$
\begin{aligned}
\#\left(M^{l^{i-1}} N / M^{l^{\prime}} N\right) & =\#\left(M^{l^{i-1}} / M^{l^{i-1}} \cap N\right) / \#\left(M^{l^{i}} / M^{l^{i}} \cap N\right) \\
& =\#\left(M^{l^{i-1}} / M^{l^{i}}\right) / \#\left(N_{i-1} / N_{i}\right) .
\end{aligned}
$$

This proves the lemma.

Proposition 1. Put $\mathscr{N}_{i}(\mathfrak{f})=k(\mathfrak{f})^{l^{i}} N_{K / k}(K(\mathfrak{f})) k_{\mathfrak{f}}$. Then

$$
\begin{aligned}
\operatorname{rank}_{i}(\mathscr{S}(K / k)) & =\operatorname{rank}_{i}(\mathrm{Cl}(k))+\operatorname{rank}_{i}\left(k(\mathfrak{f}) / N_{K / k}(K(\mathfrak{f})) k_{\mathfrak{f}}\right) \\
& +\log _{l}\left\{\#\left(F_{i-1}(\mathfrak{f}) / F_{i-1}(\mathfrak{f}) \cap \mathscr{N}_{i-1}(\mathfrak{f})\right) / \#\left(F_{i}(\mathfrak{f}) / F_{i}(\mathfrak{f}) \cap \mathscr{N}_{i}(\mathfrak{f})\right)\right\} .
\end{aligned}
$$

Proof. We apply Lemma 2 to an exact sequence

$$
\begin{aligned}
1 \rightarrow P_{k}(\mathfrak{f}) / N_{K / k} & \left(P_{K}(\mathfrak{f})\right) P_{k \mathfrak{f}} \rightarrow I_{k}(\mathfrak{f}) / N_{K / k}\left(P_{K}(\mathfrak{f})\right) P_{k \mathfrak{f}} \\
& \rightarrow I_{k}(\mathfrak{f}) / P_{k}(\mathfrak{f}) \rightarrow 1 .
\end{aligned}
$$

Then with the notations in Lemma 2 we have

$$
\begin{aligned}
N_{i} & =\left(P_{k}(F) \cap I_{k}(\mathfrak{f})^{l^{i}} N_{K / k}\left(P_{K}(\mathfrak{f})\right) P_{k \mathfrak{f}}\right) / N_{K / k}\left(P_{K}(\mathfrak{f})\right) P_{k \mathfrak{f}} \\
& \cong F_{i}(\mathfrak{f}) N_{K / k}(K(\mathfrak{f})) k_{\mathfrak{f}} / E_{k} N_{K / k}(K(\mathfrak{f})) k_{\mathfrak{f}} .
\end{aligned}
$$

Hence

$$
N_{i-1} / N_{i} \cong F_{i-1}(\mathfrak{f}) N_{K / k}(K(\mathfrak{f})) k_{\mathfrak{f}} / F_{i}(\mathfrak{f}) N_{K / k}(K(\mathfrak{f})) k_{\mathfrak{f}}
$$

So

$$
\begin{aligned}
\#\left(N_{i-1} / N_{i}\right) & =\left\{\frac{\#\left(F_{i-1}(\mathfrak{f}) N_{K / k}(K(\mathfrak{f})) k_{\mathfrak{f}} / \mathscr{N}_{i-1}(\mathfrak{f})\right)}{\#\left(F_{i}(\mathfrak{f}) N_{K / k}(K(\mathfrak{f})) k_{\mathfrak{f}} / \mathscr{N}_{i}(\mathfrak{f})\right)}\right\} \#\left(\mathscr{N}_{i-1}(\mathfrak{f}) / \mathscr{N}_{i}(\mathfrak{f})\right) \\
& =\left\{\frac{\#\left(F_{i-1}(\mathfrak{f}) / F_{i-1}(\mathfrak{f}) \cap \mathscr{N}_{i-1}(\mathfrak{f})\right)}{\#\left(F_{i}(\mathfrak{f}) / F_{i}(\mathfrak{f}) \cap \mathcal{N}_{i}(\mathfrak{f})\right)}\right\} \#\left(\mathscr{N}_{i-1}(\mathfrak{f}) / \mathscr{N}_{i}(\mathfrak{f})\right)
\end{aligned}
$$

Thus Lemma 2 implies the assertion.

Let $v$ be a prime of $k$ ramified in $K$, and let $V$ be a prime divisor of $v$ in $\bar{K}$. We also denote the restriction of $V$ to an intermediate field of $\bar{K} / k$. Let $\bar{K}_{V}, K_{V}, K_{V}^{\prime}$, and $k_{v}$ denote the completion of $\bar{K}, K, K^{\prime}$, and $k$ by $V$, respectively. Further, let $\left(K_{V}\right)^{\prime \prime}$ be the maximal abelian subextension of $\bar{K}_{V} / k_{v}$, then $K_{V}^{\prime} \subset\left(K_{V}\right)^{\prime \prime}$. Moreover, let $\left(K_{V}\right)_{2}$ be the maximal abelian subextension of $K_{V} / k_{v}$, and let $\mathfrak{f}_{V}$ and $T_{V}$ denote the conductor of $\left(K_{V}\right)_{2} / k_{v}$ and the inertia group of $v$ in $\left(K_{V}\right)_{2} / k_{v}$, respectively. Since $K / k$ is Galois, the conductor $\mathfrak{f}_{V}$ and the group $T_{V}$ do not depend on the choice of a prime divisor of $v$. Therefore, we write $\mathfrak{f}_{v}$ and $T_{v}$ instead of $\mathfrak{f}_{V}$ and $T_{V}$. On the other hand, the conductor of $\left(K_{V}\right)^{\prime \prime} / k_{v}$ coincides with $\mathrm{f}_{v}$ since $N_{\bar{K}_{V} / k_{v}}\left(U\left(\bar{K}_{V}\right)\right)=N_{K_{V} / k_{v}}\left(U\left(K_{V}\right)\right)$, where $U\left(K_{V}\right)$ denotes the group of units of $K_{V}$. Thus if we put $\mathfrak{f}^{*}=\Pi_{v} \mathfrak{f}_{v}$, then $\mathfrak{f}^{\prime} \mid \mathfrak{f}^{*}$, so we can apply the above results to $\mathfrak{f}^{*}$. 
THEOREM. Let the notation be as above. For $i \geqslant 1$, we have

$$
\begin{aligned}
\operatorname{rank}_{i}(\& S(K / k))= & \operatorname{rank}_{i}(\mathrm{Cl}(k))+\sum_{v} \operatorname{rank}_{i}\left(T_{v}\right) \\
& +\log _{l}\left\{\frac{\#\left(F_{i-1}\left(\mathfrak{f}^{*}\right) / F_{i-1}\left(\mathfrak{f}^{*}\right) \cap \mathscr{N}_{i-1}\left(\mathfrak{f}^{*}\right)\right)}{\#\left(F_{i}\left(\mathfrak{f}^{*}\right) / F_{i}\left(\mathfrak{f}^{*}\right) \cap \mathscr{N}_{i}\left(\mathfrak{f}^{*}\right)\right)}\right\} .
\end{aligned}
$$

Moreover, if $K / k$ is abelian, then $f^{*}$ is the conductor of $K / k$ and $T_{v}$ is the inertia group of $v$ in $K / k$.

Proof. We apply Proposition 1 for $f=f^{*}$. Then it suffices to prove the assertion about the second term in the right-hand side of the above formula. Clearly,

$$
k(\mathfrak{f}) / N_{K / k}(K(\mathfrak{f})) k_{\mathfrak{f}}=\prod_{v}\left(k\left(\mathfrak{f}_{v}\right) / N_{K / k}\left(K\left(\mathfrak{f}_{v}\right)\right) k_{\mathfrak{f}_{k}}\right) \quad \text { (direct) }
$$

holds. Further, $k_{\mathrm{f}_{v}} \subset N_{K_{V} / k_{v}}\left(U\left(K_{V}\right)\right)$ since $\mathfrak{f}_{v}$ is the conductor of $\left(K_{V}\right)^{\prime \prime} / k_{v}$. So we have a natural homomorphism: $k\left(\mathfrak{f}_{v}\right) / N_{K / k}\left(K\left(\mathfrak{f}_{v}\right)\right) k_{\mathfrak{f}_{v}} \rightarrow U\left(k_{v}\right) / N_{K_{V} / k_{v}}\left(U\left(K_{V}\right)\right)$; but noting $K / k$ is Galois, we can easily check that this gives an isomorphism. On the other hand, local class field theory states $U\left(k_{v}\right) / N_{K_{V} / k_{v}}\left(U\left(K_{V}\right)\right) \cong T_{v}$, which proves the theorem.

Here the last term of the above is rewritten as

$$
\log _{l}\left\{\frac{\left[\#\left(F_{i-1}(\mathfrak{f}) / k(\mathfrak{f})^{l^{i-1}}\right) / \#\left(F_{i-1}(\mathfrak{f}) \cap \mathscr{N}_{i-1}(\mathfrak{f}) / k(\mathfrak{f})^{l^{i-1}}\right)\right]}{\left[\#\left(F_{i}(\mathfrak{f}) / k(\mathfrak{f})^{l^{i}}\right) / \#\left(F_{i}(\mathfrak{f}) \cap \mathscr{N}_{i}(\mathfrak{f}) / k(\mathfrak{f})^{l^{i}}\right)\right]}\right\}
$$

hence from [3, Lemma 1] we have the following:

Corollary.

$$
\begin{aligned}
\operatorname{rank}_{i}(B S(K / k))= & \sum_{v} \operatorname{rank}_{i}\left(T_{v}\right)-\operatorname{rank}_{i}\left(E_{k}\right) \\
& +\log _{l}\left\{\frac{\#\left(F_{i}\left(\mathfrak{f}^{*}\right) \cap \mathscr{N}_{i}\left(\mathfrak{f}^{*}\right) / k\left(\mathfrak{f}^{*}\right)^{l^{i}}\right)}{\#\left(F_{i-1}\left(\mathfrak{f}^{*}\right) \cap \mathcal{N}_{i-1}\left(\mathfrak{f}^{*}\right) / k\left(\mathfrak{f}^{*}\right)^{l^{i-1}}\right)}\right\} .
\end{aligned}
$$

REMARK 1. We know for sufficiently large $i$ and $j$ (independent of $l$ ), $F_{i}\left(\mathfrak{f}^{*}\right) / F_{i}\left(\mathfrak{f}^{*}\right) \cap \mathscr{N}_{i}\left(\mathfrak{f}^{*}\right) \cong E_{k} / E_{k} \cap \mathscr{N}_{i}\left(\mathfrak{f}^{*}\right)$ and $E_{k} \cap \mathscr{N}_{i}\left(\mathfrak{f}^{*}\right)=E_{k} \cap \mathscr{N}_{j}\left(\mathfrak{f}^{*}\right)$. Moreover, taking a product for all $l$, we have $E_{k} / E_{k} \cap N_{K / k}\left(K\left(f^{*}\right)\right) k_{f^{*}} \cong$ $\Pi_{l}\left(E_{k} / E_{k} \cap \mathscr{N}^{(l)}\left(\mathfrak{f}^{*}\right)\right)$, where $\mathscr{N}^{(l)}$ denotes $\mathscr{N}_{i}$ corresponding to $l$. Thus multiplying the formulas for all $l$ and $i$ in the above theorem, we have

$$
\#(\mathfrak{S S}(K / k))=h(k) \cdot \prod_{v} \#\left(T_{v}\right) /\left[E_{k}: E_{k} \cap N_{K / k}\left(K\left(\mathrm{f}^{*}\right)\right) k_{\mathrm{f}^{*}}\right] .
$$

Let $K_{1}$ denote the maximal abelian subextension of $K / k$. Then $\left[K^{*}: K\right]=\left[K^{\prime}: K_{1}\right]$ $=\#(\&(K / k)) /\left[K_{1}: k\right]$, so the above is nothing but the genus formula (e.g. see [1]). Thus the above theorem refines the genus formula. 
REMARK 2. Now we consider an abelian case. Let $T$ be a finite set of primes of $k$. Let $M_{i}$ be the maximal abelian extension at most of index $l^{i}$ in which only primes in $T$ are ramified. For the conductor $\mathfrak{f}$ of $M_{i}$, let $M$ be the ray class field modulo $f$. Then $M_{i}$ is the maximal subfield of $M$ at most of index $l^{i}$. Hence $\log _{l} \#\left(\operatorname{Gal}\left(M_{i} / k\right)\right)=\sum_{j=1}^{i} \operatorname{rank}_{j}$ \& $(M / k)$. Put $h_{i}=\#\left\{x \in \mathrm{Cl}(k) \mid x^{i^{i}}=1\right\}$ and $t_{i}(v)=\#\left\{x \in T_{v} \mid x^{l^{i}}=1\right\}$. Then the above theorem implies

$$
\#\left(\operatorname{Gal}\left(M_{i} / k\right)\right)=h_{i}(k) \prod_{v \in T} t_{i}(v) /\left[F_{i}(\mathfrak{f}): F_{i}(\mathfrak{f}) \cap \mathscr{N}_{i}(\mathfrak{f})\right] .
$$

Since $M$ is the ray class field, $\mathscr{N}_{i}(\mathfrak{f})=k(\mathfrak{f})^{l^{i}} k_{\mathfrak{f}}$. Thus the above theorem gives a generalization of Kubota-Miki's formula [3, Theorem 1] (cf. [2]). Of course, in this case if $i=1$, then the corollary to the above theorem is [4, Theorem 1].

Finally we study the genus group of $K / k$. In general, the genus group is not determined only by $\mathbb{S}(K / k)$ and $\mathrm{Gal}(K / k)$. Indeed, as is easily seen there are abelian fields $K / Q$ and $L / Q$ such that $\operatorname{Gal}(K / Q) \cong \operatorname{Gal}(L / Q)$ and $K^{*}=L^{*}$ although $\operatorname{Gal}\left(K^{*} / K\right)$ is not isomorphic to $\operatorname{Gal}\left(L^{*} / L\right)$. However, in the following case the genus group is completely determined by $\mathbb{S}(K / k)$ and $\mathrm{Gal}\left(K_{1} / k\right)$ since $\operatorname{Gal}\left(K^{*} / K\right) \cong \operatorname{Gal}\left(K^{\prime} / K_{1}\right)$. The proof of Proposition 2 is easy, so we omit it.

Proposition 2. If there exists a finite set $T$ of primes of $k$ such that $\operatorname{Gal}\left(K_{1} / k\right)=$ $\Pi_{v \in T} T_{v}$ (direct product), then

$$
\text { (S) }(K / k) \cong \operatorname{Gal}\left(K_{1} / k\right) \oplus \operatorname{Gal}\left(K^{\prime} / K_{1}\right) \text {. }
$$

REMARK 3. In this paper we deal with the wide sense, but a similar argument holds in the narrow sense with a few changes of parts about infinite primes.

\section{REFERENCES}

1. Y. Furuta, The genus field and genus number in algebraic number fields, Nagoya Math. J. 29 (1967), $281-285$.

2. T. Kubota, Galois group of the maximal abelian extension over an algebraic number field, Nagoya Math. J. 12 (1957), 177-189.

3. H. Miki, On the maximal abelian l-extension of a finite algebraic number field with given ramification, Nagoya Math. J. 70 (1978), 183-202.

4. I. R. Šfarevič, Extensions with given points of ramification, Inst. Hautes Études Sci. Publ. Math. 18 (1963), 71-95; Amer. Math. Soc. Transl. 59 (1966), 128-149.

Department of Mathematics, Faculty of General Education, Niggata University, Nigata $950-21$, JAPAN 